\title{
Addendum
}

to the paper:

\section{Finite Waiting Space Bulk Service System*}

\section{P. SINGH}

\section{Special Cases:}

(I) $M / G^{s} / 1 /(N+1)$ System:

This is a "bulk service" system and the "service capacity" in this case is fixed. Thus we have

$$
b_{r}=\left\{\begin{array}{lll}
1 & \text { for } & r=0 \\
0 & \text { for } & r \neq 0
\end{array}\right.
$$

and hence $B_{s-i}=1=B_{s-i}(x)=B_{s}(x)$.

Therefore the expression (17) for $Q(x)$ reduces to

$$
Q(x)=\frac{\sum_{i=0}^{s-1} p_{i}\left(x^{s}-x^{i}\right)}{x^{s} / K(x)-1} .
$$

The expression corresponding to (6) for $p_{N}$ is

$$
p_{N}=\frac{\sum_{r=0}^{N-s-1} l_{N-r} p_{s+r}+l_{N}\left(\sum_{i=0}^{s} p_{i}\right)}{\left(1-l_{s}\right)}
$$

(II) $E_{\mathrm{s}} / G / 1 /(N+1)$ System:

In this case the inter-arrival time has the distribution

$$
e^{-\lambda x} \frac{\lambda^{s} x^{s-1}}{(s-1) !} d x
$$

Following Bhat [2] if we consider an input process which is Poisson with parameter $\lambda$, then the interval between the arrival points of consecutive $s^{\text {th }}$ customers also has the distribution $\left(^{*}\right)$ above; and therefore instead of each customer of the system $E_{s} / G / 1$ we can think of $s$ hypothetical customers who arrive in a Poisson process and get served in a single batch. Consequently the study of $E_{s} / G / 1$ system is identical with that of $M / G^{s} / 1$ system and the results (18) and (19) above are valid in this case.

If $Q_{n}^{\prime}$ is the queue length just after the $n^{\text {th }}$ departure in the system $E_{s} / G / 1 /(N+1)$ and $Q_{n}$ be that in $M / G^{s} / 1 /(N+1)$, then $Q_{n}^{\prime}$ may be obtained from $Q_{n}$ by using the relationship

$$
Q_{n}^{\prime}=\left[\frac{Q_{n}}{s}\right]
$$

where $[\cdot]$ is the largest integer in the argument.

* Journal of Engineering Mathematics, Volume 5, nr. 4, pp. 241-247, October 1971. 
(III) $M^{r} / G^{s} / 1 /(N+1)$ System:

This is a bulk queueing system, where arrivals are in groups of size $r$ and service is in groups of size $s$. In this case the expression for $k_{j}$ becomes

$$
k_{j}=\left\{\begin{array}{l}
\int_{0}^{\infty} e^{-\lambda t} \frac{(\lambda t)^{j / r}}{(j / r) !} d G(t) \text { for } j=m r, m=0,1,2, \ldots \\
0 \quad \text { otherwise }
\end{array}\right.
$$

The analysis of this system is similar to that of the $M / G^{s} / 1 /(N+1)$ system with minor changes in the transition probability matrix.

(IV) $M / G / 1 /(N+1)$ System:

In this case the service capacity $s$ is one. The expression for $P(x)$ now becomes

$$
P(x)=\frac{p_{0}(1-x) K(x)}{K(x)-x}
$$

The expression determining $p_{N}$ is

$$
p_{N}=\frac{\sum_{r=0}^{N} l_{N-r} p_{r+1}+l_{N} p_{0}}{1-l_{1}} .
$$

An Alternative Approach:

In this case the expressions for $p_{j}$ 's from (4) can be written explicitely as follows:

$$
\begin{aligned}
p_{0} & =p_{0} k_{0}+p_{1} k_{0} \\
p_{1} & =p_{0} k_{1}+p_{1} k_{1}+p_{2} k_{0} \\
p_{2} & =p_{0} k_{2}+p_{1} k_{2}+p_{2} k_{1}+p_{3} k_{0} \\
p_{3} & =p_{0} k_{3}+p_{1} k_{3}+p_{2} k_{2}+p_{3} k_{1}+p_{4} k_{0} \\
& \vdots \\
p_{n-1} & =p_{0} k_{n-1}+p_{1} k_{n-1}+p_{2} k_{n-2}+\ldots+p_{n-1} k_{1}+p_{n} k_{0},
\end{aligned}
$$

Note that we do not need the last equation for $p_{n}$, since the last column of the transition probability matrix depends on the previous ones. These equations can be solved resursively.

Let $A_{j}=p_{j} / p_{0}$, then since from the first equation $A_{0}+A_{1}=1 / k_{0}$, the solution is given by

$$
\begin{aligned}
\Lambda_{0} & =1 \\
\Lambda_{1} k_{0} & =\left(1-k_{0}\right) \\
\Lambda_{2} k_{0} & =\Lambda_{1}\left(1-k_{1}\right)-k_{1} \\
A_{3} k_{0} & =\Lambda_{2}\left(1-k_{1}\right)-\frac{k_{2}}{k_{0}} \\
\Lambda_{4} k_{0} & =\Lambda_{3}\left(1-k_{1}\right)-\Lambda_{2} k_{2}-\frac{k_{3}}{k_{0}} \\
\Lambda_{5} k_{0} & =\Lambda_{4}\left(1-k_{1}\right)-\Lambda_{3} k_{2}-\Lambda_{2} k_{3}-\frac{k_{4}}{k_{0}} \\
& \vdots \vdots \vdots \\
\Lambda_{n} k_{0} & =\Lambda_{n-1}\left(1-k_{1}\right)-\Lambda_{n-2} k_{2}-\Lambda_{n-3} k_{3} \ldots-\Lambda_{2} k_{n-2}-\frac{k_{n-1}}{k_{0}} .
\end{aligned}
$$


Now the solution $p_{j}$ satisfying (7) and $\Sigma p_{j}=1$ is given by

$$
p_{j}=\frac{\Lambda_{j}}{\sum_{i=0}^{N} \Lambda_{i}}, \quad j \leqq N
$$

(V) $M / M / 1 /(N+1)$ System:

In this case the service times have negative exponential distribution with parameter $\mu$ (say), i.e.,

$$
g(t)=\mu e^{-\mu t}, \quad t \geqq 0
$$

therefore

$$
k_{j}=\left(\frac{\mu}{\lambda+\mu}\right)^{j}\left(\frac{\mu}{\lambda+\mu}\right)
$$

Then following the arguments in Singh [8] we have

$$
p_{j}=\left\{\begin{array}{lll}
\frac{(1-\rho) \rho^{j}}{1-\rho^{N+1}} & \rho<1 \quad j=0,1,2, \ldots, N \\
\frac{1}{N+1} & \rho=1 & \text { where } \quad \rho=\frac{\lambda}{\mu}
\end{array}\right.
$$

In this case the average number of units in the system is

$$
E(Q)= \begin{cases}\rho \cdot \sqrt{\left.\frac{1-(N+1) \rho^{N}+N \rho^{N+1}}{(1-\rho)\left(1-\rho^{N+1}\right)}\right],}, & \rho<1 \\ \frac{N}{2} & \rho=1\end{cases}
$$

The average time an arrival spends in the system is:

$$
E(W)= \begin{cases}\frac{\rho}{\mu}\left[\frac{1-(N+1) \rho^{N}+N \rho^{N+1}}{(1-\rho)\left(1-\rho^{N+1}\right)}\right], & \rho<1 \\ \frac{N}{2 \mu} & \rho=1\end{cases}
$$

(VI) $M / D / 1 /(N+1)$ System:

In this case

$$
g(t)=\delta\left(t-\frac{1}{\mu}\right)=\left\{\begin{array}{l}
1 \text { for } t=\frac{1}{\mu} \\
0 \text { for } t \neq \frac{1}{\mu}
\end{array}\right.
$$

and

$$
k_{j}=e^{-\rho} \frac{\rho^{j}}{j !} \text { where } \rho=\frac{\lambda}{\mu} .
$$

Using the same arguments as in Singh [8], we have

$$
p_{j}=\left[\sum_{r=1}^{j} \frac{e^{-\rho r}(-\rho r)^{j-r}}{(j-r) !}-\sum_{r=1}^{j-1} \frac{e^{\rho r}(-\rho r)^{j-r-1}}{(j-r-1) !}\right] p_{0}
$$

where $p_{0}$ is given by the following expression 


$$
p_{0}=\sum_{j=0}^{N} \sum_{r=1}^{j} \frac{e^{\rho r}(-\rho r)^{j-r}}{(j-r) !}-\left.\sum_{j=0}^{N} \sum_{r=1}^{j-1} \frac{e^{\rho r}(-\rho r)^{j-r-1}}{(j-r-1) !}\right|^{-1} .
$$

In this case, the average number of units in the system is given by

$$
E(Q)=\left|\sum_{j=1}^{N} \sum_{r=1}^{j} \frac{j e^{\rho r}(-\rho r)^{j-r}}{(j-r) !}-\sum_{j=1}^{N} \sum_{r=1}^{j-1} j \cdot \frac{e^{\rho r}(-\rho r)^{j-r-1}}{(j-r-1) !}\right| p_{0} .
$$

We remark that the evaluation of $p_{j}$ 's in principle is a straight forward problem of power series expansion, yet for service times other than negative exponential, involves heavy algebraic computations. This fact is quite obviously demonstrated by the expression for $p_{j}$ 's in the $M / D / 1$ system.

Journal of Engineering Math., Vol. 6 (1972) 85-88 